\title{
Combined effect of body mass index and body size perception on metabolic syndrome in South Korea: results of the fifth Korea National Health and Nutrition Examination Surveys (2010-2012)
}

Sook Hee Yoon ${ }^{1+}$, Kyu-Tae Han ${ }^{2,3+}$, Sun Jung Kim ${ }^{4}$,Tae Yong Sohn ${ }^{5}$, Byungyool Jeon ${ }^{6}$, Woorim Kim²,3 and Eun-Cheol Park ${ }^{6^{*}}$

\begin{abstract}
Background: Body mass index (BMI) has been used as an indirect predictor for the risk of metabolic syndrome. However, there are challenges in evaluating the risk of metabolic syndrome using BMI in certain parts of the world. Therefore, it is worth exploring additional factors that could supplement BMI to predict the risk of metabolic syndrome. In this study, we assessed the combined effect of BMI and perception for predicting metabolic syndrome.

Methods: We used the fifth Korea National Health and Nutrition Examination Surveys (KNHANES V, 2010-12, $N=16,537)$ in this study. Multivariable logistic regression analysis was performed to examine the association while controlling for potential confounding variables. We also performed an analysis for the combined effect of BMI and perception of body size, and subgroup analysis by age group or moderate physical activity.

Results: Data from 16,537 participants were analyzed in this study (males: 6,978, females: 9,559). Among them, metabolic syndrome was diagnosed in 1,252 (17.9\%) males and 2,445 (25.6\%) females, respectively. The combination of BMI and body size perception had a positive relation with the presence of metabolic syndrome. People who perceived themselves to be overweight for their body size had a higher risk for metabolic syndrome even if they have the same BMI.
\end{abstract}

Conclusion: Our findings suggest that the combination of body size perception and BMI is useful in predicting the risk of metabolic syndrome. The use of complementary predictors could reduce the risk for inaccurate prediction of metabolic syndrome.

Keywords: Metabolic syndrome, Body mass index, BMI, Perception of body size, Combined effect

\section{Background}

South Korea has achieved rapid socioeconomic development since the late $20^{\text {th }}$ century. This fast-paced growth has led to changes in South Koreans' daily lives, affecting lifestyle and food consumption, and contributing to improved overall health status as South Korea becomes an aging society $[1,2]$. However, there has been a concomitant

\footnotetext{
* Correspondence: ecpark@yuhs.ac

†Equal contributors

${ }^{6}$ Department of Preventive Medicine, Yonsei University College of Medicine, Seoul, Republic of Korea

Full list of author information is available at the end of the article
}

increase in new health problems in South Korea, such as higher rates of chronic disease. According to Statistics Korea, cardiovascular diseases were the fifth leading cause of death in South Korea (50.2 deaths per 100,000 people in 2013) [3].

In the 2013 Organization for Economic Co-operation and Development (OECD) Health at Glance report, South Korea compares poorly with other OECD countries [4]. This problem is expected to be exacerbated by an aging population. To solve those problems, many health care professionals have studied chronic diseases and identified metabolic syndrome as a major cause $[5,6]$. Metabolic

\section{Ciomed Central}


syndrome has rapidly increased in South Korea over the past few decades (1998 year: $24.9 \%$, 2007 year: $31.3 \%$ ) [7]. Problems related with metabolic syndrome are expected to continue to increase. Thus, preventing metabolic syndrome is important for managing chronic diseases.

Metabolic syndrome is generally diagnosed by five indicators: waist circumference, triglyceride level, high-density lipoprotein (HDL) cholesterol level, blood pressure, and fasting glucose level. If three indicators (including waist circumference) are met, an individual is diagnosed with metabolic syndrome [8]. Many previous studies identified obesity as the major risk factor of metabolic syndrome. Thus, body mass index (BMI) has been widely used as an indirect predictor for evaluating the risk of metabolic syndrome $[9,10]$. However, the use of BMI to predict metabolic syndrome is not necessarily applicable in every country; this simple metric does not consider important factors such as racial/ethnic differences and lifestyle factors. Even in people with the same BMI, the risk of metabolic syndrome may differ, depending on whether they smoke or consume alcohol [11-13]. Therefore, it is worth exploring additional predictors that could supplement BMI to assess the risk of metabolic syndrome; here, we focus on body size perception.

Although many previous studies have assessed the relationship between body size perception and obesity, few have also investigated the incidence of metabolic syndrome in South Korea [14, 15]. Perception of body size is a factor that affects peoples' lifestyle, including food consumption. Moreover, the risk of metabolic syndrome can be changed by altering one's lifestyle. In this study, we analyzed the relationship between the incidence of metabolic syndrome and BMI or body size perception, as well as the combined effect of BMI and the body size perception on metabolic syndrome.

\section{Methods \\ Study population}

This study used data from the fifth Korea National Health and Nutrition Examination Surveys (KNHANES V, 2010-12). KNHANES are cross-sectional surveys that have been conducted annually since 1998 by the Korea Centers for Disease Control and Prevention (KCDC) to assess the health and nutritional status of the Korean population. A stratified multistage cluster-sampling design was used to obtain a nationally representative sample. This survey is composed of three parts: Health Interview Survey, Health Examination, and Nutrition Survey. We used data from the Health Interview Survey, Health Examination, and Nutrition Survey. The overall response rates were $81.9 \%$ in 2010, 80.4 \% in 2011, and $80.0 \%$ in 2012 . A total of 25,967 individuals (8,958 in 2010, 8,491 in 2011, and 8,518 in 2012) completed the survey. Any respondents who did not provide BMI, perceptions of body size, five indicators for the diagnosis of metabolic syndrome, or were under the age of 19 were excluded from the study. We ultimately included 14,773 eligible participants in this study. The KNHANES was openly available in https:// knhanes.cdc.go.kr/knhanes/eng/index.do after submitting e-mail address and registering short-form information. These data was approved by the KCDC Institutional Review Board, and all participants provided written informed consent (2010-02CON-21-C, 2011-02CON-06-C, 2012-01-EXP-01-2C).

\section{Variables}

The outcome variable in this study was the incidence of metabolic syndrome, which was defined by the International Diabetes Federation (IDF) criteria. It was diagnosed if two of the five indicators (including waist circumference) met the IDF criteria for waist circumference, triglyceride level, HDL cholesterol level, blood pressure, and fasting glucose level.

IDF criteria (metabolic syndrome diagnosed if two or more indicators were present)

1. Waist circumference (male: $\geq 90 \mathrm{~cm}$ and female: $\geq 80 \mathrm{~cm}$ for Asian subjects)

2. Triglycerides level $(\geq 150 \mathrm{mg} / \mathrm{dl})$

3. HDL cholesterol level (male: $<40 \mathrm{mg} / \mathrm{dl}$, female: $<50 \mathrm{mg} / \mathrm{dl}$ )

4. Blood pressure (systolic: $\geq 130 \mathrm{mmHg}$, diastolic: $\geq 85 \mathrm{mmHg}$, or treatment of diagnosed hypertension)

5. Fasting glucose level ( $\geq 100 \mathrm{mg} / \mathrm{dl}$ or type 2 diabetes)

The independent variables of main interest in relation to metabolic syndrome were BMI and body size perception. BMI was calculated as body weight $(\mathrm{kg})$ divided into the squared height $\left(\mathrm{m}^{2}\right)$. BMI was classified into three groups as follows: $\leq 22.9,23.0-24.9$, or $\geq 25$. Perception of body size was defined as the answer to the question: "How do you perceive your body size?" The response to this question was classified into: underweight, normal, or overweight.

Other independent variables considered in analysis as potential confounding variables were age, sex, income, educational level, economic activity, marital status, sleep duration, smoking status, alcohol consumption, stress awareness, moderate physical activity, menopause (only female), total energy intake and survey year. Income status was classified as "low", "mid-low", "mid-high", or "high". Economic activity was defined as "yes" or "no". Stress awareness was classified as "high", "moderate", or "low". Moderate physical activity was defined as whether respondents performed moderate physical activity for 30 min per session more than 5 times per week. Total energy intake was calculated based on respondent's selfreported for their usual food consumption. 


\section{Statistical analysis}

We first examined the distribution of each categorical variable by frequency and percentages and performed $x^{2}$ tests to identify correlation with combination of BMI and body size perception by sex. Next, we performed analysis of variance (ANOVA) for continuous variables as total energy intake to identify correlation with combination of BMI and body size perception and to compare average and standard deviation of variables. In addition, these analyses were also performed to examine differences in each variable according to incidence of metabolic syndrome by sex. Multivariable logistic regression analysis was used to examine the association between BMI or body size perception and metabolic syndrome while controlling for potential confounding variables such as age, sex, income, educational level, economic activity, marital status, sleep duration, smoking status, alcohol consumption, stress awareness, moderate physical activity, menopause (only female), total energy intake, and survey year. We also included menopause status for female respondents. An additional analysis was carried out for the combined effect of BMI and body size perception, as was subgroup analysis by either age group ( $<$ vs. $\geq 65$ years) or physical activity. Sampling weights assigned to each participant were applied in the analyses to generalize the sampled data. C-statistics were calculated to examine the predictive values for the logistics model. These values range between 0 (no predictive value) and 1, (perfect predictive value). All statistical analyses were performed using SAS statistical software (Cary, NC) version 9.2.

\section{Results}

Data from 14,773 participants were analyzed in this study (males: 5,897, females: 8,876). Tables 1 and 2 shows the association between combination of BMI and body size perception and other covariates by sex. Among them, people who rightly perceived their body size were as follows: males = BMI, $\leq 22.9,52.6 \%$; 23.0-24.9, $64.7 \%$; $\geq 25,78.1 \%$, and females = BMI, $\leq 22.9,26.2 \% ; 23.0-24.9$, $38.0 \%$; $\geq 25,82.5 \%$. There were statistically significant correlations with combination of BMI and body size perception in both sex $(\mathrm{P}<.0001)$. By the results of association between combination of BMI and body size perception and covariates, most of covariates had statistically significant correlations with variables of interest, except to moderate physical activity and survey year in males; moderate physical activity and menopause in females.

Table 3 shows the univariate associations between each variable and metabolic syndrome. Among them, metabolic syndrome was noted in 1,062 (18.0\%) males and 2,304 (26.0 \%) females. In both males and females, higher BMI were more frequent in those with metabolic syndrome (males: $\leq 22.9,1.5 \%$; 23.0-24.9, $11.1 \%$; $\geq 25$, $42.1 \%$ and females: $\leq 22.9,5.3 \%$; 23.0-24.9, $28.5 \%$; $\geq 25$, $56.4 \%)$. By body size perception, people who responded overweight were more frequently determined to have metabolic syndrome regardless of sex (males: underweight, $2.0 \%$; normal, $9.9 \%$; overweight, $37.3 \%$ and females: underweight, $12.7 \%$; normal, $18.7 \%$; overweight, $36.7 \%$ ). In addition, males who overestimated their body size than BMI were more frequent in those with metabolic syndrome, but females who underestimated their body size were more frequent in those with metabolic syndrome.

The older age group had a higher rate of female metabolic syndrome. Notably, the distribution for metabolic syndrome had an inverse relationship with income in females (low, 42.4 \%; mid-low, $28.0 \%$; mid-high, $20.1 \%$; high, $16.4 \%)$. Similarly, subjects who were separated, widowed, or divorced were more likely to meet the criteria for metabolic syndrome compared to those with other marital statuses (males: married, $19.2 \%$; separated/widowed/divorced, $19.9 \%$; single, $7.1 \%$ and females: married, $23.7 \%$; separated/widowed/divorced, $43.7 \%$; single, $5.2 \%$; Table 3 ).

Table 4 shows the results of logistic regression analysis for the association between BMI and metabolic syndrome adjusted for covariates by sex. In both males and females, BMI had a positive relationship with metabolic syndrome (males: $\leq 22.9=$ ref, $23.0-24.9$ odds ratio [OR]: 9.17, standard deviation [SD]: 5.81-14.50; $\geq 25$ OR: 71.08, SD: 46.32-109.08; females: $\leq 22.9=$ ref, $23.0-24.9=$ OR: 6.79, SD: $5.57-8.28, \geq 25=$ OR: 27.75, SD: 22.71-33.91). Age also had a positive relationship with metabolic syndrome, whereas educational level only had an inverse relationship with metabolic syndrome in females. Both sexes who did not report economic activity had a higher risk for metabolic syndrome (males: yes $=$ ref, $n o=O R: 1.50, S D=$ 1.10-2.05; females: no $=\mathrm{OR}: 1.27, \mathrm{SD}=1.08-1.48$ ), as did smokers of males. Females who had experienced menopause had a higher risk for metabolic syndrome (not yet $=$ ref, yes = OR: 1.46, SD = 1.09-1.94; Table 4).

Table 5 shows the logistic regression analysis results for the association between combined effect of BMI/ body size perception and metabolic syndrome adjusted for covariates by sex. The combination of BMI and body size perception had a positive relationship with metabolic syndrome. People who perceived themselves as overweight for their body size had a higher risk for metabolic syndrome, even if they had the same BMI as a person who did not consider themselves overweight. The results of other controlling variables had similar values and trends as the results listed in Table 4 (Table 5).

We also performed subgroup analysis for the combined effect of BMI/body size perception by age group (< vs. $\geq 65$ years) or moderate physical activity to identify possible differences in each group. In the subgroup 
Table 1 Association between combination of BMI and perception of body size and covariates in male

\begin{tabular}{|c|c|c|c|c|c|c|c|c|c|c|c|c|c|c|c|c|c|c|c|}
\hline \multirow{4}{*}{$\begin{array}{l}\frac{\text { BMI }}{\text { Perception of body size }} \\
\text { Variables }\end{array}$} & \multicolumn{19}{|c|}{ Males $(n=5,897)$} \\
\hline & \multicolumn{6}{|c|}{$\leq 22.9(n=2,340)$} & \multicolumn{6}{|c|}{$23.0-24.9(n=1,520)$} & \multicolumn{6}{|c|}{$\geq 25(n=2,037)$} & \multirow[t]{3}{*}{ P-value } \\
\hline & \multicolumn{2}{|c|}{$\begin{array}{l}\text { Underweight } \\
(52.6 \%)\end{array}$} & \multicolumn{2}{|l|}{$\begin{array}{l}\text { Normal } \\
(44.0 \%)\end{array}$} & \multicolumn{2}{|c|}{$\begin{array}{l}\text { Overweight } \\
(3.3 \%)\end{array}$} & \multicolumn{2}{|c|}{$\begin{array}{l}\text { Underweight } \\
(5.3 \%)\end{array}$} & \multicolumn{2}{|l|}{$\begin{array}{l}\text { Normal } \\
(64.7 \%)\end{array}$} & \multicolumn{2}{|c|}{$\begin{array}{l}\text { Overweight } \\
(30.0 \%)\end{array}$} & \multicolumn{2}{|c|}{$\begin{array}{l}\text { Underweight } \\
(1.1 \%)\end{array}$} & \multicolumn{2}{|l|}{$\begin{array}{l}\text { Normal } \\
(20.9 \%)\end{array}$} & \multicolumn{2}{|c|}{$\begin{array}{l}\text { Overweight } \\
(78.1 \%)\end{array}$} & \\
\hline & N/Mean & $\% / S D$ & N/Mean & $\% / S D$ & N/Mean & $\% / S D$ & N/Mean & $\% / S D$ & N/Mean & $\% / S D$ & N/Mean & $\% / S D$ & N/Mean & $\% / S D$ & N/Mean & $\% / S D$ & N/Mean & $\% / S D$ & \\
\hline \multicolumn{20}{|l|}{ Age (years) } \\
\hline $19 \sim 29$ & 119 & 26.6 & 91 & 20.4 & 14 & 3.1 & 1 & 0.2 & 51 & 11.4 & 36 & 8.1 & 0 & 0.0 & 9 & 2.0 & 126 & 28.2 & $<.0001$ \\
\hline $30 \sim 39$ & 186 & 19.9 & 144 & 15.4 & 13 & 1.4 & 5 & 0.5 & 130 & 13.9 & 83 & 8.9 & 1 & 0.1 & 37 & 4.0 & 336 & 35.9 & \\
\hline $40 \sim 49$ & 178 & 17.3 & 136 & 13.2 & 15 & 1.5 & 10 & 1.0 & 169 & 16.4 & 79 & 7.7 & 1 & 0.1 & 72 & 7.0 & 368 & 35.8 & \\
\hline $50 \sim 59$ & 211 & 18.1 & 171 & 14.7 & 8 & 0.7 & 13 & 1.1 & 239 & 20.5 & 123 & 10.5 & 2 & 0.2 & 92 & 7.9 & 307 & 26.3 & \\
\hline $60 \sim 69$ & 247 & 20.4 & 215 & 17.8 & 13 & 1.1 & 23 & 1.9 & 216 & 17.9 & 87 & 7.2 & 10 & 0.8 & 110 & 9.1 & 289 & 23.9 & \\
\hline$\geq 70$ & 291 & 26.2 & 273 & 24.6 & 15 & 1.4 & 28 & 2.5 & 179 & 16.1 & 48 & 4.3 & 8 & 0.7 & 105 & 9.5 & 164 & 14.8 & \\
\hline \multicolumn{20}{|l|}{ Income } \\
\hline Low & 306 & 27.0 & 235 & 20.7 & 22 & 1.9 & 31 & 2.7 & 181 & 15.9 & 57 & 5.0 & 6 & 0.5 & 106 & 9.3 & 191 & 16.8 & $<.0001$ \\
\hline Mid-low & 364 & 23.5 & 273 & 17.6 & 20 & 1.3 & 14 & 0.9 & 262 & 16.9 & 90 & 5.8 & 7 & 0.5 & 117 & 7.6 & 400 & 25.9 & \\
\hline Mid-high & 295 & 18.3 & 283 & 17.6 & 18 & 1.1 & 21 & 1.3 & 276 & 17.2 & 135 & 8.4 & 6 & 0.4 & 98 & 6.1 & 477 & 29.6 & \\
\hline High & 267 & 16.6 & 239 & 14.9 & 18 & 1.1 & 14 & 0.9 & 265 & 16.5 & 174 & 10.8 & 3 & 0.2 & 104 & 6.5 & 522 & 32.5 & \\
\hline \multicolumn{20}{|l|}{ Educational level } \\
\hline $\begin{array}{l}\text { Below elementary } \\
\text { school }\end{array}$ & 297 & 24.9 & 262 & 22.0 & 20 & 1.7 & 34 & 2.9 & 205 & 17.2 & 59 & 5.0 & 8 & 0.7 & 125 & 10.5 & 181 & 15.2 & $<.0001$ \\
\hline $\begin{array}{l}\text { Middle school } \\
\text { graduated }\end{array}$ & 158 & 20.8 & 116 & 15.2 & 5 & 0.7 & 16 & 2.1 & 141 & 18.5 & 55 & 7.2 & 4 & 0.5 & 78 & 10.2 & 188 & 24.7 & \\
\hline $\begin{array}{l}\text { High school } \\
\text { graduated }\end{array}$ & 426 & 21.2 & 352 & 17.6 & 23 & 1.1 & 14 & 0.7 & 331 & 16.5 & 162 & 8.1 & 6 & 0.3 & 128 & 6.4 & 563 & 28.1 & \\
\hline $\begin{array}{l}\text { Above University } \\
\text { graduated }\end{array}$ & 351 & 18.1 & 300 & 15.5 & 30 & 1.5 & 16 & 0.8 & 307 & 15.8 & 180 & 9.3 & 4 & 0.2 & 94 & 4.8 & 658 & 33.9 & \\
\hline \multicolumn{20}{|l|}{ Economic activity } \\
\hline Yes & 870 & 20.0 & 709 & 16.3 & 49 & 1.1 & 45 & 1.0 & 716 & 16.5 & 356 & 8.2 & 13 & 0.3 & 314 & 7.2 & 1,273 & 29.3 & $<.0001$ \\
\hline No & 362 & 23.3 & 321 & 20.7 & 29 & 1.9 & 35 & 2.3 & 268 & 17.3 & 100 & 6.4 & 9 & 0.6 & 111 & 7.2 & 317 & 20.4 & \\
\hline \multicolumn{20}{|l|}{ Marital status } \\
\hline Married & 1,012 & 20.2 & 840 & 16.8 & 62 & 1.2 & 70 & 1.4 & 861 & 17.2 & 391 & 7.8 & 21 & 0.4 & 383 & 7.7 & 1,361 & 27.2 & $<.0001$ \\
\hline $\begin{array}{l}\text { Separated/ } \\
\text { Bereavement/ } \\
\text { Divorced }\end{array}$ & 64 & 20.9 & 64 & 20.9 & 3 & 1.0 & 8 & 2.6 & 57 & 18.6 & 18 & 5.9 & 1 & 0.3 & 27 & 8.8 & 64 & 20.9 & \\
\hline Single & 156 & 26.4 & 126 & 21.4 & 13 & 2.2 & 2 & 0.3 & 66 & 11.2 & 47 & 8.0 & 0 & 0.0 & 15 & 2.5 & 165 & 28.0 & \\
\hline
\end{tabular}


Table 1 Association between combination of BMI and perception of body size and covariates in male (Continued)

\begin{tabular}{|c|c|c|c|c|c|c|c|c|c|c|c|c|c|c|c|c|c|c|c|}
\hline \multicolumn{20}{|l|}{ Sleep duration } \\
\hline Less than $6 \mathrm{~h}$ & 497 & 20.5 & 408 & 16.8 & 35 & 1.4 & 36 & 1.5 & 401 & 16.5 & 206 & 8.5 & 14 & 0.6 & 167 & 6.9 & 660 & 27.2 & 0.0025 \\
\hline $7-8 \mathrm{~h}$ & 644 & 21.0 & 520 & 17.0 & 36 & 1.2 & 39 & 1.3 & 517 & 16.9 & 222 & 7.2 & 6 & 0.2 & 230 & 7.5 & 852 & 27.8 & \\
\hline More than $9 \mathrm{~h}$ & 91 & 22.4 & 102 & 25.1 & 7 & 1.7 & 5 & 1.2 & 66 & 16.2 & 28 & 6.9 & 2 & 0.5 & 28 & 6.9 & 78 & 19.2 & \\
\hline \multicolumn{20}{|l|}{ Smoking status } \\
\hline $\begin{array}{l}\text { Non-smoker/ } \\
\text { Ex-smoker }\end{array}$ & 704 & 19.2 & 648 & 17.6 & 49 & 1.3 & 56 & 1.5 & 635 & 17.3 & 273 & 7.4 & 17 & 0.5 & 300 & 8.2 & 992 & 27.0 & $<.0001$ \\
\hline Smoker & 528 & 23.8 & 382 & 17.2 & 29 & 1.3 & 24 & 1.1 & 349 & 15.7 & 183 & 8.2 & 5 & 0.2 & 125 & 5.6 & 598 & 26.9 & \\
\hline \multicolumn{20}{|l|}{ Alcohol consumption } \\
\hline Never & 246 & 23.1 & 219 & 20.5 & 21 & 2.0 & 26 & 2.4 & 181 & 17.0 & 59 & 5.5 & 9 & 0.8 & 92 & 8.6 & 213 & 20.0 & $<.0001$ \\
\hline $\begin{array}{l}\text { Less than } 1 \text { time } \\
\text { per month }\end{array}$ & 259 & 23.4 & 197 & 17.8 & 10 & 0.9 & 13 & 1.2 & 197 & 17.8 & 81 & 7.3 & 4 & 0.4 & 63 & 5.7 & 284 & 25.6 & \\
\hline $\begin{array}{l}\text { Less than } 3 \text { times } \\
\text { per week }\end{array}$ & 530 & 18.5 & 460 & 16.1 & 39 & 1.4 & 32 & 1.1 & 456 & 15.9 & 254 & 8.9 & 8 & 0.3 & 187 & 6.5 & 895 & 31.3 & \\
\hline $\begin{array}{l}\text { More than } 4 \text { times } \\
\text { per week }\end{array}$ & 197 & 22.9 & 154 & 17.9 & 8 & 0.9 & 9 & 1.0 & 150 & 17.4 & 62 & 7.2 & 1 & 0.1 & 83 & 9.6 & 198 & 23.0 & \\
\hline \multicolumn{20}{|l|}{ Stress awareness } \\
\hline High & 298 & 23.4 & 179 & 14.1 & 20 & 1.6 & 20 & 1.6 & 185 & 14.5 & 118 & 9.3 & 0 & 0.0 & 70 & 5.5 & 384 & 30.1 & $<.0001$ \\
\hline Moderate & 724 & 20.8 & 608 & 17.4 & 45 & 1.3 & 41 & 1.2 & 580 & 16.6 & 272 & 7.8 & 15 & 0.4 & 250 & 7.2 & 950 & 27.3 & \\
\hline Low & 210 & 18.5 & 243 & 21.4 & 13 & 1.1 & 19 & 1.7 & 219 & 19.2 & 66 & 5.8 & 7 & 0.6 & 105 & 9.2 & 256 & 22.5 & \\
\hline \multicolumn{20}{|l|}{$\begin{array}{l}\text { Moderate physical } \\
\text { activity }\end{array}$} \\
\hline No & 1,115 & 20.8 & 924 & 17.3 & 75 & 1.4 & 70 & 1.3 & 889 & 16.6 & 413 & 7.7 & 21 & 0.4 & 383 & 7.2 & 1,460 & 27.3 & 0.4056 \\
\hline Yes & 117 & 21.4 & 106 & 19.4 & 3 & 0.5 & 10 & 1.8 & 95 & 17.4 & 43 & 7.9 & 1 & 0.2 & 42 & 7.7 & 130 & 23.8 & \\
\hline \multicolumn{20}{|l|}{ Survey year } \\
\hline 2010 & 396 & 21.1 & 313 & 16.6 & 32 & 1.7 & 28 & 1.5 & 311 & 16.5 & 145 & 7.7 & 4 & 0.2 & 148 & 7.9 & 504 & 26.8 & 0.5417 \\
\hline 2011 & 456 & 21.4 & 374 & 17.6 & 25 & 1.2 & 25 & 1.2 & 341 & 16.0 & 166 & 7.8 & 8 & 0.4 & 136 & 6.4 & 595 & 28.0 & \\
\hline 2012 & 380 & 20.1 & 343 & 18.1 & 21 & 1.1 & 27 & 1.4 & 332 & 17.6 & 145 & 7.7 & 10 & 0.5 & 141 & 7.5 & 491 & 26.0 & \\
\hline Total energy intake & $2,312.9$ & \pm 906.0 & $2,252.5$ & \pm 903.6 & $2,269.2$ & $\pm 1,033.1$ & $2,073.1$ & \pm 894.1 & $2,362.6$ & \pm 895.1 & $2,321.0$ & \pm 924.7 & $1,980.4$ & \pm 713.5 & $2,360.0$ & $\pm 1,113.0$ & $2,461.1$ & \pm 963.6 & $<.0001$ \\
\hline Total & 1,232 & 20.9 & 1,030 & 17.5 & 78 & 1.3 & 80 & 1.4 & 984 & 16.7 & 456 & 7.7 & 22 & 0.4 & 425 & 7.2 & 1,590 & 27.0 & \\
\hline
\end{tabular}

BMI body mass index 
Table 2 Association between combination of BMI and perception of body size and covariates in female

\begin{tabular}{|c|c|c|c|c|c|c|c|c|c|c|c|c|c|c|c|c|c|c|c|}
\hline \multirow{4}{*}{$\begin{array}{l}\text { BMI } \\
\text { Perception of body size } \\
\text { Variables }\end{array}$} & \multicolumn{19}{|c|}{ Females $(n=8,876)$} \\
\hline & \multicolumn{6}{|c|}{$\leq 22.9(n=4,219)$} & \multicolumn{6}{|c|}{$23.0-24.9(n=1,956)$} & \multicolumn{6}{|c|}{$\geq 25(n=2,701)$} & \multirow[t]{3}{*}{ P-value } \\
\hline & \multicolumn{2}{|c|}{$\begin{array}{l}\text { Underweight } \\
(26.2 \%)\end{array}$} & \multicolumn{2}{|l|}{$\begin{array}{l}\text { Normal } \\
(57.6 \%)\end{array}$} & \multicolumn{2}{|c|}{$\begin{array}{l}\text { Overweight } \\
(16.3 \%)\end{array}$} & \multicolumn{2}{|c|}{$\begin{array}{l}\text { Underweight } \\
(5.7 \%)\end{array}$} & \multicolumn{2}{|l|}{$\begin{array}{l}\text { Normal } \\
(38.0 \%)\end{array}$} & \multicolumn{2}{|c|}{$\begin{array}{l}\text { Overweight } \\
(56.3 \%)\end{array}$} & \multicolumn{2}{|c|}{$\begin{array}{l}\text { Underweight } \\
(2.6 \%)\end{array}$} & \multicolumn{2}{|l|}{$\begin{array}{l}\text { Normal } \\
(14.9 \%)\end{array}$} & \multicolumn{2}{|c|}{$\begin{array}{l}\text { Overweight } \\
(82.5 \%)\end{array}$} & \\
\hline & N/Mean & $\% / S D$ & N/Mean & $\% / S D$ & N/Mean & $\% / S D$ & N/Mean & $\% / S D$ & N/Mean & $\% / S D$ & N/Mean & $\% / S D$ & N/Mean & $\% / S D$ & N/Mean & $\% / S D$ & N/Mean & $\% / S D$ & \\
\hline \multicolumn{20}{|l|}{ Age (years) } \\
\hline $19 \sim 29$ & 135 & 18.1 & 293 & 39.3 & 116 & 15.5 & 0 & 0.0 & 15 & 2.0 & 67 & 9.0 & 0 & 0.0 & 1 & 0.1 & 119 & 16.0 & $<.0001$ \\
\hline $30 \sim 39$ & 183 & 10.7 & 659 & 38.5 & 265 & 15.5 & 0 & 0.0 & 46 & 2.7 & 231 & 13.5 & 0 & 0.0 & 12 & 0.7 & 314 & 18.4 & \\
\hline $40 \sim 49$ & 140 & 8.7 & 515 & 31.9 & 149 & 9.2 & 2 & 0.1 & 75 & 4.6 & 286 & 17.7 & 0 & 0.0 & 18 & 1.1 & 430 & 26.6 & \\
\hline $50 \sim 59$ & 163 & 9.1 & 446 & 24.9 & 104 & 5.8 & 6 & 0.3 & 153 & 8.5 & 294 & 16.4 & 6 & 0.3 & 52 & 2.9 & 569 & 31.7 & \\
\hline $60 \sim 69$ & 181 & 11.8 & 270 & 17.6 & 31 & 2.0 & 26 & 1.7 & 227 & 14.8 & 156 & 10.2 & 20 & 1.3 & 126 & 8.2 & 494 & 32.3 & \\
\hline$\geq 70$ & 302 & 20.4 & 246 & 16.6 & 21 & 1.4 & 78 & 5.3 & 227 & 15.3 & 67 & 4.5 & 44 & 3.0 & 194 & 13.1 & 302 & 20.4 & \\
\hline \multicolumn{20}{|l|}{ Income } \\
\hline Low & 329 & 17.2 & 364 & 19.0 & 57 & 3.0 & 70 & 3.7 & 240 & 12.5 & 128 & 6.7 & 43 & 2.2 & 195 & 10.2 & 487 & 25.5 & $<.0001$ \\
\hline Mid-low & 252 & 11.0 & 587 & 25.7 & 164 & 7.2 & 19 & 0.8 & 202 & 8.8 & 297 & 13.0 & 12 & 0.5 & 115 & 5.0 & 640 & 28.0 & \\
\hline Mid-high & 232 & 9.9 & 746 & 31.8 & 236 & 10.1 & 11 & 0.5 & 148 & 6.3 & 287 & 12.2 & 9 & 0.4 & 58 & 2.5 & 618 & 26.4 & \\
\hline High & 291 & 12.5 & 732 & 31.4 & 229 & 9.8 & 12 & 0.5 & 153 & 6.6 & 389 & 16.7 & 6 & 0.3 & 35 & 1.5 & 483 & 20.7 & \\
\hline \multicolumn{20}{|l|}{ Educational level } \\
\hline $\begin{array}{l}\text { Below elementary } \\
\text { school }\end{array}$ & 455 & 15.4 & 492 & 16.7 & 65 & 2.2 & 103 & 3.5 & 417 & 14.1 & 229 & 7.8 & 63 & 2.1 & 314 & 10.6 & 811 & 27.5 & $<.0001$ \\
\hline $\begin{array}{l}\text { Middle school } \\
\text { graduated }\end{array}$ & 94 & 9.9 & 205 & 21.6 & 38 & 4.0 & 5 & 0.5 & 109 & 11.5 & 131 & 13.8 & 5 & 0.5 & 44 & 4.6 & 316 & 33.4 & \\
\hline $\begin{array}{l}\text { High school } \\
\text { graduated }\end{array}$ & 254 & 9.3 & 833 & 30.4 & 300 & 11.0 & 4 & 0.1 & 151 & 5.5 & 420 & 15.3 & 1 & 0.0 & 33 & 1.2 & 741 & 27.1 & \\
\hline $\begin{array}{l}\text { Above University } \\
\text { graduated }\end{array}$ & 301 & 13.4 & 899 & 40.1 & 283 & 12.6 & 0 & 0.0 & 66 & 2.9 & 321 & 14.3 & 1 & 0.0 & 12 & 0.5 & 360 & 16.0 & \\
\hline \multicolumn{20}{|l|}{ Economic activity } \\
\hline Yes & 493 & 12.0 & 1,166 & 28.3 & 318 & 7.7 & 41 & 1.0 & 320 & 7.8 & 562 & 13.6 & 16 & 0.4 & 154 & 3.7 & 1,048 & 25.4 & $<.0001$ \\
\hline No & 611 & 12.8 & 1,263 & 26.5 & 368 & 7.7 & 71 & 1.5 & 423 & 8.9 & 539 & 11.3 & 54 & 1.1 & 249 & 5.2 & 1,180 & 24.8 & \\
\hline \multicolumn{20}{|l|}{ Marital status } \\
\hline Married & 708 & 10.8 & 1,832 & 28.1 & 540 & 8.3 & 50 & 0.8 & 534 & 8.2 & 894 & 13.7 & 34 & 0.5 & 232 & 3.6 & 1,704 & 26.1 & $<.0001$ \\
\hline $\begin{array}{l}\text { Separated/Bereavement/ } \\
\text { Divorced }\end{array}$ & 266 & 16.1 & 319 & 19.3 & 44 & 2.7 & 62 & 3.7 & 198 & 12.0 & 136 & 8.2 & 36 & 2.2 & 170 & 10.3 & 425 & 25.7 & \\
\hline Single & 130 & 18.8 & 278 & 40.2 & 102 & 14.7 & 0 & 0.0 & 11 & 1.6 & 71 & 10.3 & 0 & 0.0 & 1 & 0.1 & 99 & 14.3 & \\
\hline
\end{tabular}


Table 2 Association between combination of BMl and perception of body size and covariates in female (Continued)

\begin{tabular}{|c|c|c|c|c|c|c|c|c|c|c|c|c|c|c|c|c|c|c|c|}
\hline \multicolumn{20}{|l|}{ Sleep duration } \\
\hline Less than $6 \mathrm{~h}$ & 469 & 12.5 & 933 & 24.9 & 220 & 5.9 & 59 & 1.6 & 358 & 9.6 & 451 & 12.0 & 39 & 1.0 & 220 & 5.9 & 999 & 26.7 & $<.0001$ \\
\hline $7-8 \mathrm{~h}$ & 530 & 12.0 & 1,311 & 29.6 & 398 & 9.0 & 37 & 0.8 & 328 & 7.4 & 592 & 13.4 & 23 & 0.5 & 149 & 3.4 & 1,061 & 24.0 & \\
\hline More than $9 \mathrm{~h}$ & 105 & 15.0 & 185 & 26.5 & 68 & 9.7 & 16 & 2.3 & 57 & 8.2 & 58 & 8.3 & 8 & 1.1 & 34 & 4.9 & 168 & 24.0 & \\
\hline \multicolumn{20}{|l|}{ Smoking status } \\
\hline Non-smoker/Ex-smoker & 1,043 & 12.3 & 2,312 & 27.3 & 642 & 7.6 & 109 & 1.3 & 726 & 8.6 & 1,051 & 12.4 & 69 & 0.8 & 394 & 4.7 & 2,126 & 25.1 & 0.0019 \\
\hline Smoker & 61 & 15.1 & 117 & 29.0 & 44 & 10.9 & 3 & 0.7 & 17 & 4.2 & 50 & 12.4 & 1 & 0.2 & 9 & 2.2 & 102 & 25.2 & \\
\hline \multicolumn{20}{|l|}{ Alcohol consumption } \\
\hline Never & 526 & 15.1 & 843 & 24.2 & 164 & 4.7 & 67 & 1.9 & 346 & 9.9 & 365 & 10.5 & 50 & 1.4 & 235 & 6.8 & 885 & 25.4 & $<.0001$ \\
\hline $\begin{array}{l}\text { Less than } 1 \\
\text { time per month }\end{array}$ & 367 & 11.3 & 923 & 28.3 & 287 & 8.8 & 27 & 0.8 & 252 & 7.7 & 448 & 13.7 & 15 & 0.5 & 107 & 3.3 & 833 & 25.6 & \\
\hline $\begin{array}{l}\text { Less than } 3 \\
\text { times per week }\end{array}$ & 188 & 9.5 & 622 & 31.5 & 223 & 11.3 & 15 & 0.8 & 126 & 6.4 & 270 & 13.7 & 4 & 0.2 & 54 & 2.7 & 472 & 23.9 & \\
\hline $\begin{array}{l}\text { More than } 4 \\
\text { times per week }\end{array}$ & 23 & 14.2 & 41 & 25.3 & 12 & 7.4 & 3 & 1.9 & 19 & 11.7 & 18 & 11.1 & 1 & 0.6 & 7 & 4.3 & 38 & 23.5 & \\
\hline \multicolumn{20}{|l|}{ Stress awareness } \\
\hline High & 360 & 14.6 & 637 & 25.8 & 225 & 9.1 & 40 & 1.6 & 164 & 6.6 & 287 & 11.6 & 26 & 1.1 & 84 & 3.4 & 648 & 26.2 & $<.0001$ \\
\hline Moderate & 558 & 11.0 & 1,474 & 29.1 & 398 & 7.9 & 41 & 0.8 & 425 & 8.4 & 682 & 13.5 & 24 & 0.5 & 197 & 3.9 & 1,263 & 25.0 & \\
\hline Low & 186 & 13.8 & 318 & 23.7 & 63 & 4.7 & 31 & 2.3 & 154 & 11.5 & 132 & 9.8 & 20 & 1.5 & 122 & 9.1 & 317 & 23.6 & \\
\hline \multicolumn{20}{|l|}{$\begin{array}{l}\text { Moderate physical } \\
\text { activity }\end{array}$} \\
\hline No & 1,027 & 12.6 & 2,253 & 27.6 & 635 & 7.8 & 102 & 1.3 & 679 & 8.3 & 1,012 & 12.4 & 61 & 0.7 & 369 & 4.5 & 2,013 & 24.7 & 0.0755 \\
\hline Yes & 77 & 10.6 & 176 & 24.3 & 51 & 7.0 & 10 & 1.4 & 64 & 8.8 & 89 & 12.3 & 9 & 1.2 & 34 & 4.7 & 215 & 29.7 & \\
\hline \multicolumn{20}{|l|}{ Survey year } \\
\hline 2010 & 330 & 11.8 & 752 & 26.8 & 222 & 7.9 & 38 & 1.4 & 227 & 8.1 & 382 & 13.6 & 21 & 0.7 & 125 & 4.5 & 708 & 25.2 & $<.0001$ \\
\hline 2011 & 416 & 13.3 & 863 & 27.5 & 238 & 7.6 & 39 & 1.2 & 257 & 8.2 & 360 & 11.5 & 24 & 0.8 & 148 & 4.7 & 792 & 25.2 & \\
\hline 2012 & 358 & 12.2 & 814 & 27.7 & 226 & 7.7 & 35 & 1.2 & 259 & 8.8 & 359 & 12.2 & 25 & 0.9 & 130 & 4.4 & 728 & 24.8 & \\
\hline \multicolumn{20}{|l|}{ Menopause } \\
\hline Not yet & 464 & 11.1 & 1,499 & 35.8 & 534 & 12.8 & 3 & 0.1 & 152 & 3.6 & 599 & 14.3 & 0 & 0.0 & 34 & 0.8 & 898 & 21.5 & 0.7918 \\
\hline Yes & 640 & 13.6 & 930 & 19.8 & 152 & 3.2 & 109 & 2.3 & 591 & 12.6 & 502 & 10.7 & 70 & 1.5 & 369 & 7.9 & 1,330 & 28.3 & \\
\hline Total energy intake & $1,683.0$ & \pm 649.9 & $1,747.8$ & \pm 656.5 & $1,724.0$ & \pm 699.4 & $1,447.7$ & \pm 608.8 & $1,629.2$ & \pm 576.5 & $1,683.4$ & \pm 654.6 & $1,412.0$ & \pm 423.2 & $1,610.2$ & \pm 643.0 & $1,694.6$ & \pm 650.1 & $<.0001$ \\
\hline Total & 1,104 & 12.4 & 2,429 & 27.4 & 686 & 7.7 & 112 & 1.3 & 743 & 8.4 & 1,101 & 12.4 & 70 & 0.8 & 403 & 4.5 & 2,228 & 25.1 & \\
\hline
\end{tabular}

BMI body mass index 
Table 3 Demographic characteristics by metabolic syndrome (frequency, \%)

\begin{tabular}{|c|c|c|c|c|c|c|c|c|c|c|c|}
\hline \multirow{4}{*}{ Variables } & & \multicolumn{10}{|c|}{ Metabolic syndrome $(n=14,773)$} \\
\hline & & \multicolumn{5}{|c|}{ Males $(n=5,897)$} & \multicolumn{5}{|c|}{ Females $(n=8,876)$} \\
\hline & & \multicolumn{2}{|l|}{ Yes } & \multicolumn{2}{|l|}{ No } & \multirow[t]{2}{*}{ P-value } & \multicolumn{2}{|l|}{ Yes } & \multicolumn{2}{|l|}{ No } & \multirow[t]{2}{*}{ P-value } \\
\hline & & N/Mean & $\% / S D$ & N/Mean & $\% / S D$ & & N/Mean & $\% / S D$ & N/Mean & $\% / S D$ & \\
\hline \multicolumn{12}{|l|}{ BMl } \\
\hline$\leq 22.9$ & & 36 & 1.5 & 2,304 & 98.5 & $<.0001$ & 222 & 5.3 & 3,997 & 94.7 & $<.0001$ \\
\hline $23.0-24.9$ & & 168 & 11.1 & 1,352 & 88.9 & & 558 & 28.5 & 1,398 & 71.5 & \\
\hline$\geq 25$ & & 858 & 42.1 & 1,179 & 57.9 & & 1,524 & 56.4 & 1,177 & 43.6 & \\
\hline \multicolumn{12}{|l|}{ Perception of body size } \\
\hline Underweight & & 27 & 2.0 & 1,307 & 98.0 & $<.0001$ & 163 & 12.7 & 1,123 & 87.3 & $<.0001$ \\
\hline Normal & & 242 & 9.9 & 2,197 & 90.1 & & 667 & 18.7 & 2,908 & 81.3 & \\
\hline Overweight & & 793 & 37.3 & 1,331 & 62.7 & & 1,474 & 36.7 & 2,541 & 63.3 & \\
\hline BMI & Perception of body size & & & & & & & & & & \\
\hline \multirow[t]{3}{*}{$\leq 22.9$} & Underweight & 11 & 0.9 & 1,221 & 99.1 & $<.0001$ & 63 & 5.7 & 1,041 & 94.3 & $<.0001$ \\
\hline & Normal & 20 & 1.9 & 1,010 & 98.1 & & 131 & 5.4 & 2,298 & 94.6 & \\
\hline & Overweight & 5 & 6.4 & 73 & 93.6 & & 28 & 4.1 & 658 & 95.9 & \\
\hline \multirow[t]{3}{*}{$23.0-24.9$} & Underweight & 10 & 12.5 & 70 & 87.5 & & 53 & 47.3 & 59 & 52.7 & \\
\hline & Normal & 90 & 9.1 & 894 & 90.9 & & 267 & 35.9 & 476 & 64.1 & \\
\hline & Overweight & 68 & 14.9 & 388 & 85.1 & & 238 & 21.6 & 863 & 78.4 & \\
\hline \multirow[t]{3}{*}{$\geq 25$} & Underweight & 6 & 27.3 & 16 & 72.7 & & 47 & 67.1 & 23 & 32.9 & \\
\hline & Normal & 132 & 31.1 & 293 & 68.9 & & 269 & 66.7 & 134 & 33.3 & \\
\hline & Overweight & 720 & 45.3 & 870 & 54.7 & & 1,208 & 54.2 & 1,020 & 45.8 & \\
\hline \multicolumn{12}{|l|}{ Age (years) } \\
\hline $19 \sim 29$ & & 23 & 5.1 & 424 & 94.9 & $<.0001$ & 26 & 3.5 & 720 & 96.5 & $<.0001$ \\
\hline $30 \sim 39$ & & 126 & 13.5 & 809 & 86.5 & & 113 & 6.6 & 1,597 & 93.4 & \\
\hline $40 \sim 49$ & & 186 & 18.1 & 842 & 81.9 & & 256 & 15.9 & 1,359 & 84.1 & \\
\hline $50 \sim 59$ & & 229 & 19.6 & 937 & 80.4 & & 486 & 27.1 & 1,307 & 72.9 & \\
\hline $60 \sim 69$ & & 275 & 22.7 & 935 & 77.3 & & 689 & 45.0 & 842 & 55.0 & \\
\hline$\geq 70$ & & 223 & 20.1 & 888 & 79.9 & & 734 & 49.6 & 747 & 50.4 & \\
\hline \multicolumn{12}{|l|}{ Income } \\
\hline Low & & 211 & 18.6 & 924 & 81.4 & 0.7869 & 812 & 42.4 & 1,101 & 57.6 & $<.0001$ \\
\hline Mid-low & & 275 & 17.8 & 1,272 & 82.2 & & 640 & 28.0 & 1,648 & 72.0 & \\
\hline Mid-high & & 279 & 17.3 & 1,330 & 82.7 & & 471 & 20.1 & 1,874 & 79.9 & \\
\hline High & & 297 & 18.5 & 1,309 & 81.5 & & 381 & 16.4 & 1,949 & 83.6 & \\
\hline \multicolumn{12}{|l|}{ Educational level } \\
\hline Below elementary school & & 212 & 17.8 & 979 & 82.2 & $<.0001$ & 1,363 & 46.2 & 1,586 & 53.8 & $<.0001$ \\
\hline Middle school graduated & & 186 & 24.4 & 575 & 75.6 & & 308 & 32.5 & 639 & 67.5 & \\
\hline High school graduated & & 337 & 16.8 & 1,668 & 83.2 & & 457 & 16.7 & 2,280 & 83.3 & \\
\hline Above University graduated & & 327 & 16.9 & 1,613 & 83.1 & & 176 & 7.8 & 2,067 & 92.2 & \\
\hline \multicolumn{12}{|l|}{ Economic activity } \\
\hline Yes & & 761 & 17.5 & 3,584 & 82.5 & 0.0980 & 905 & 22.0 & 3,213 & 78.0 & $<.0001$ \\
\hline No & & 301 & 19.4 & 1,251 & 80.6 & & 1,399 & 29.4 & 3,359 & 70.6 & \\
\hline \multicolumn{12}{|l|}{ Marital status } \\
\hline Married & & 959 & 19.2 & 4,042 & 80.8 & $<.0001$ & 1,544 & 23.7 & 4,984 & 76.3 & $<.0001$ \\
\hline
\end{tabular}


Table 3 Demographic characteristics by metabolic syndrome (frequency, \%) (Continued)

\begin{tabular}{|c|c|c|c|c|c|c|c|c|c|c|}
\hline $\begin{array}{l}\text { Separated/Bereavement/ } \\
\text { Divorced }\end{array}$ & 61 & 19.9 & 245 & 80.1 & & 724 & 43.7 & 932 & 56.3 & \\
\hline Single & 42 & 7.1 & 548 & 92.9 & & 36 & 5.2 & 656 & 94.8 & \\
\hline \multicolumn{11}{|l|}{ Sleep duration } \\
\hline Less than $6 \mathrm{~h}$ & 439 & 18.1 & 1,985 & 81.9 & 0.9858 & 1,133 & 30.2 & 2,615 & 69.8 & $<.0001$ \\
\hline $7-8 \mathrm{~h}$ & 550 & 17.9 & 2,516 & 82.1 & & 973 & 22.0 & 3,456 & 78.0 & \\
\hline More than $9 \mathrm{~h}$ & 73 & 17.9 & 334 & 82.1 & & 198 & 28.3 & 501 & 71.7 & \\
\hline \multicolumn{11}{|l|}{ Smoking status } \\
\hline Non-smoker/Ex-smoker & 683 & 18.6 & 2,991 & 81.4 & 0.1356 & 2,215 & 26.1 & 6,257 & 73.9 & 0.0653 \\
\hline Smoker & 379 & 17.0 & 1,844 & 83.0 & & 89 & 22.0 & 315 & 78.0 & \\
\hline \multicolumn{11}{|l|}{ Alcohol consumption } \\
\hline Never & 185 & 17.4 & 881 & 82.6 & $<.0001$ & 1,177 & 33.8 & 2,304 & 66.2 & $<.0001$ \\
\hline Less than 1 time per month & 154 & 13.9 & 954 & 86.1 & & 721 & 22.1 & 2,538 & 77.9 & \\
\hline Less than 3 times per week & 520 & 18.2 & 2,341 & 81.8 & & 365 & 18.5 & 1,609 & 81.5 & \\
\hline More than 4 times per week & 203 & 23.5 & 659 & 76.5 & & 41 & 25.3 & 121 & 74.7 & \\
\hline \multicolumn{11}{|l|}{ Stress awareness } \\
\hline High & 221 & 17.3 & 1,053 & 82.7 & 0.0139 & 622 & 25.2 & 1,849 & 74.8 & $<.0001$ \\
\hline Moderate & 602 & 17.3 & 2,883 & 82.7 & & 1,210 & 23.9 & 3,852 & 76.1 & \\
\hline Low & 239 & 21.0 & 899 & 79.0 & & 472 & 35.1 & 871 & 64.9 & \\
\hline \multicolumn{11}{|l|}{ Moderate physical activity } \\
\hline No & 982 & 18.4 & 4,368 & 81.6 & 0.0306 & 2,102 & 25.8 & 6,049 & 74.2 & 0.2222 \\
\hline Yes & 80 & 14.6 & 467 & 85.4 & & 202 & 27.9 & 523 & 72.1 & \\
\hline \multicolumn{11}{|l|}{ Survey year } \\
\hline 2010 & 360 & 19.1 & 1,521 & 80.9 & 0.0240 & 737 & 26.3 & 2,068 & 73.7 & 0.1602 \\
\hline 2011 & 399 & 18.8 & 1,727 & 81.2 & & 778 & 24.8 & 2,359 & 75.2 & \\
\hline 2012 & 303 & 16.0 & 1,587 & 84.0 & & 789 & 26.9 & 2,145 & 73.1 & \\
\hline \multicolumn{11}{|l|}{ Menopause } \\
\hline Not yet & - & - & - & - & - & 417 & 10.0 & 3,766 & 90.0 & $<.0001$ \\
\hline Yes & - & - & - & - & & 1,887 & 40.2 & 2,806 & 59.8 & \\
\hline Total energy intake & $2,365.6$ & \pm 970.4 & $2,346.0$ & \pm 935.0 & 0.5391 & $1,619.0$ & \pm 640.1 & $1,720.2$ & \pm 651.7 & $<.0001$ \\
\hline Total & 1,062 & 18.0 & 4,835 & 82.0 & & 2,304 & 26.0 & 6,572 & 74.0 & \\
\hline
\end{tabular}

$B M I$ body mass index

analysis by age group, it revealed similar relationships of the combined effect of BMI and body size perception in these two groups as were observed in the overall analysis. However, there were some notable findings in nonelderly females. In the overweight group based on BMI, the risk for metabolic syndrome was inversely associated with body size perception (Table 6). In the results of subgroup analysis by moderate physical activity, overweight or obese people based on BMI who underestimated their body size had a higher trend regarding the risk of metabolic syndrome in the moderate physical activity of over 5 times per week group than the other group (data not shown). In the overall multivariable logistic regression, $\mathrm{C}$-statistics were higher in the combination model of BMI and body size perception than when using only BMI models.

\section{Discussion}

Due to the rapidly aging population, it is expected that the prevalence of metabolic syndrome will continue to increase in South Korea [16]. It is therefore necessary to design effective strategies to prevent and manage this chronic condition. In recent years, BMI has become a widely used indicator of obesity and indirect predictor for metabolic syndrome. However, it had some limitations that were not overall considered to risk factors for metabolic syndrome $[17,18]$. Thus, it is necessary to find complementary predictive factors; we focused on body size perception as a novel predictor for evaluating metabolic syndrome risk. Our results suggest that metabolic syndrome risk was positively related with BMI and were similar to previous studies that examined metabolic syndrome risk factors. 
Table 4 Results of multivariable logistic regression analysis for the relationship between BMI and metabolic syndrome

\begin{tabular}{|c|c|c|c|c|c|c|}
\hline \multirow{3}{*}{ Variables } & \multicolumn{6}{|c|}{ Metabolic syndrome } \\
\hline & \multicolumn{3}{|l|}{ Males } & \multicolumn{3}{|c|}{ Females } \\
\hline & $\overline{\mathrm{OR}}$ & SD & & $\overline{\mathrm{OR}}$ & SD & \\
\hline \multicolumn{7}{|l|}{$\mathrm{BMI}$} \\
\hline$\leq 22.9$ & 1.00 & - & - & 1.00 & - & - \\
\hline $23.0-24.9$ & 9.17 & 5.81 & 14.50 & 6.79 & 5.57 & 8.28 \\
\hline$\geq 25$ & 71.08 & 46.32 & 109.08 & 27.75 & 22.71 & 33.91 \\
\hline \multicolumn{7}{|l|}{ Age (years) } \\
\hline $19 \sim 29$ & 1.00 & - & - & 1.00 & - & - \\
\hline $30 \sim 39$ & 2.21 & 1.17 & 4.17 & 2.16 & 1.18 & 3.96 \\
\hline $40 \sim 49$ & 3.05 & 1.67 & 5.58 & 4.31 & 2.38 & 7.79 \\
\hline $50 \sim 59$ & 4.20 & 2.19 & 8.04 & 5.02 & 2.58 & 9.77 \\
\hline $60 \sim 69$ & 5.45 & 2.69 & 11.02 & 8.47 & 4.27 & 16.81 \\
\hline$\geq 70$ & 7.01 & 3.41 & 14.44 & 12.11 & 6.28 & 23.35 \\
\hline \multicolumn{7}{|l|}{ Income } \\
\hline Low & 1.00 & - & - & 1.00 & - & - \\
\hline Mid-low & 1.18 & 0.84 & 1.66 & 1.07 & 0.87 & 1.33 \\
\hline Mid-high & 1.12 & 0.80 & 1.58 & 0.98 & 0.77 & 1.23 \\
\hline High & 1.29 & 0.90 & 1.84 & 0.87 & 0.67 & 1.14 \\
\hline \multicolumn{7}{|l|}{ Educational level } \\
\hline Below elementary school & 1.00 & - & - & 1.00 & - & - \\
\hline Middle school graduated & 1.25 & 0.90 & 1.74 & 0.71 & 0.56 & 0.91 \\
\hline High school graduated & 0.98 & 0.72 & 1.33 & 0.59 & 0.46 & 0.76 \\
\hline Above University graduated & 1.03 & 0.73 & 1.43 & 0.51 & 0.37 & 0.72 \\
\hline \multicolumn{7}{|l|}{ Economic activity } \\
\hline Yes & 1.00 & - & - & 1.00 & - & - \\
\hline No & 1.50 & 1.10 & 2.05 & 1.27 & 1.08 & 1.48 \\
\hline \multicolumn{7}{|l|}{ Marital status } \\
\hline Married & 1.00 & - & - & 1.00 & - & - \\
\hline Separated/Bereavement/Divorced & 0.81 & 0.52 & 1.26 & 1.10 & 0.92 & 1.31 \\
\hline Single & 0.85 & 0.53 & 1.38 & 1.26 & 0.69 & 2.28 \\
\hline \multicolumn{7}{|l|}{ Sleep duration } \\
\hline Less than $6 \mathrm{~h}$ & 0.91 & 0.75 & 1.10 & 0.85 & 0.72 & 1.00 \\
\hline $7-8 h$ & 1.00 & - & - & 1.00 & - & - \\
\hline More than $9 \mathrm{~h}$ & 0.85 & 0.55 & 1.32 & 1.14 & 0.87 & 1.51 \\
\hline \multicolumn{7}{|l|}{ Smoking status } \\
\hline Non-smoker/Ex-smoker & 1.00 & - & - & 1.00 & - & - \\
\hline Smoker & 1.31 & 1.04 & 1.64 & 1.37 & 0.92 & 2.06 \\
\hline \multicolumn{7}{|l|}{ Alcohol consumption } \\
\hline Never & 1.00 & - & - & 1.00 & - & - \\
\hline Less than 1 time per month & 1.03 & 0.73 & 1.46 & 0.96 & 0.80 & 1.16 \\
\hline Less than 3 times per week & 1.16 & 0.85 & 1.59 & 1.00 & 0.80 & 1.24 \\
\hline More than 4 times per week & 1.92 & 1.36 & 2.72 & 0.84 & 0.49 & 1.43 \\
\hline
\end{tabular}


Table 4 Results of multivariable logistic regression analysis for the relationship between BMI and metabolic syndrome (Continued)

\begin{tabular}{|c|c|c|c|c|c|c|}
\hline \multicolumn{7}{|l|}{ Stress awareness } \\
\hline High & 0.99 & 0.70 & 1.38 & 0.90 & 0.70 & 1.17 \\
\hline Moderate & 0.90 & 0.69 & 1.18 & 0.85 & 0.68 & 1.07 \\
\hline Low & 1.00 & - & - & 1.00 & - & - \\
\hline \multicolumn{7}{|c|}{ Moderate physical activity } \\
\hline No & 1.00 & - & - & 1.00 & - & - \\
\hline Yes & 1.29 & 0.89 & 1.87 & 1.09 & 0.84 & 1.41 \\
\hline \multicolumn{7}{|l|}{ Survey year } \\
\hline 2010 & 1.00 & - & - & 1.00 & - & - \\
\hline 2011 & 1.14 & 0.90 & 1.45 & 0.89 & 0.74 & 1.08 \\
\hline 2012 & 0.82 & 0.63 & 1.07 & 1.14 & 0.95 & 1.37 \\
\hline \multicolumn{7}{|l|}{ Menopause } \\
\hline Not yet & - & - & - & 1.00 & - & - \\
\hline Yes & - & - & - & 1.45 & 1.09 & 1.92 \\
\hline Total energy intake & 1.00 & 0.99 & 1.01 & 1.01 & 0.99 & 1.02 \\
\hline C-statistics & $0.855^{*}$ & & & $0.876^{*}$ & & \\
\hline
\end{tabular}

$B M I$ body mass index, $O R$ odds ratio, $S D$, standard deviation

*P-value for likelihood ratio test $<0.05$

In addition, we observed a combined effect of body size perception and BMI on the risk of metabolic syndrome. Notably, the risk was clearer than that observed using BMI only, and was even observed in subjects with the same BMI but different body perceptions.

In predicting risk for chronic diseases as metabolic syndrome, using only BMI could make some misidentifications because it was calculated by just considering height and weight. If people had same BMI, the risk for metabolic syndrome could be different by major factors consisted of body constitution such as muscle mass and higher body fat [19]. Therefore, using combination of BMI and body size perception would be more helpful in predicting for risk. Based on our results, perception of body size as overweight had higher risk for metabolic syndrome. This is because that perception of body size as overweight could more reflect to risk for metabolic syndrome considering actual body image in same BMI. Perception of body size can help role of complementation of predicting for metabolic syndrome [20]. Therefore, it is suggested that people who perceive their body size as overweight are likely to be at risk of metabolic syndrome. In another point of view, people could respond as overweight for their body size due to their unhealthy behaviors such as unhealthy diet and insufficient physical activity for preventing chronic diseases even if people with same BMI and similar body constitution [21]. Therefore, perception of body size could be indirect indicators for reflecting life styles as well as actual body image.

The same phenomenon was observed when we performed a subgroup analysis by age group that excluded females who were overweight based on BMI and $<65$ years. This relationship was more positive in males, while the different results in females $<65$ years may be caused by younger females who did not exhibit health behaviors such as wrong diet and insufficient exercise due to their misperception for their body size despite being overweight or obesity based on BMI. However, in the case of elderly females, they had an effort to manage their health status due to their health concern by advanced age [22]. Based on the results of the subgroup analysis in the moderate physical activity group, people overweight or obese based on BMI tend to exhibit unhealthy behaviors by underestimating their body size and risks of gaining metabolic syndrome as they show moderate physical activity. They may be overconfident, believing in an improvement of their health status by sufficient physical activity, and could take more risky behaviors such as excessive eating. Therefore, providing correct information about preventing metabolic syndrome would be needed.

Although more detailed studies are needed, our findings suggest that inappropriate perception of their health status could be caused to unhealthy behaviors at risky population. This has been described previously; people who are borderline for chronic disease risk do not usually feel that their lives are at risk [23]. Conversely, highrisk populations were much more amenable to health behaviors to modify their risk. It is important to note that males tend to evaluate their own body status more favorably than females. Perception differences can induce people to make lifestyle changes (e.g., food or alcohol consumption, exercise, smoking status, etc.) [15, 24, 25]. 
Table 5 Results of multivariable logistic regression analysis for the relationship between BMI/body size perception and metabolic syndrome

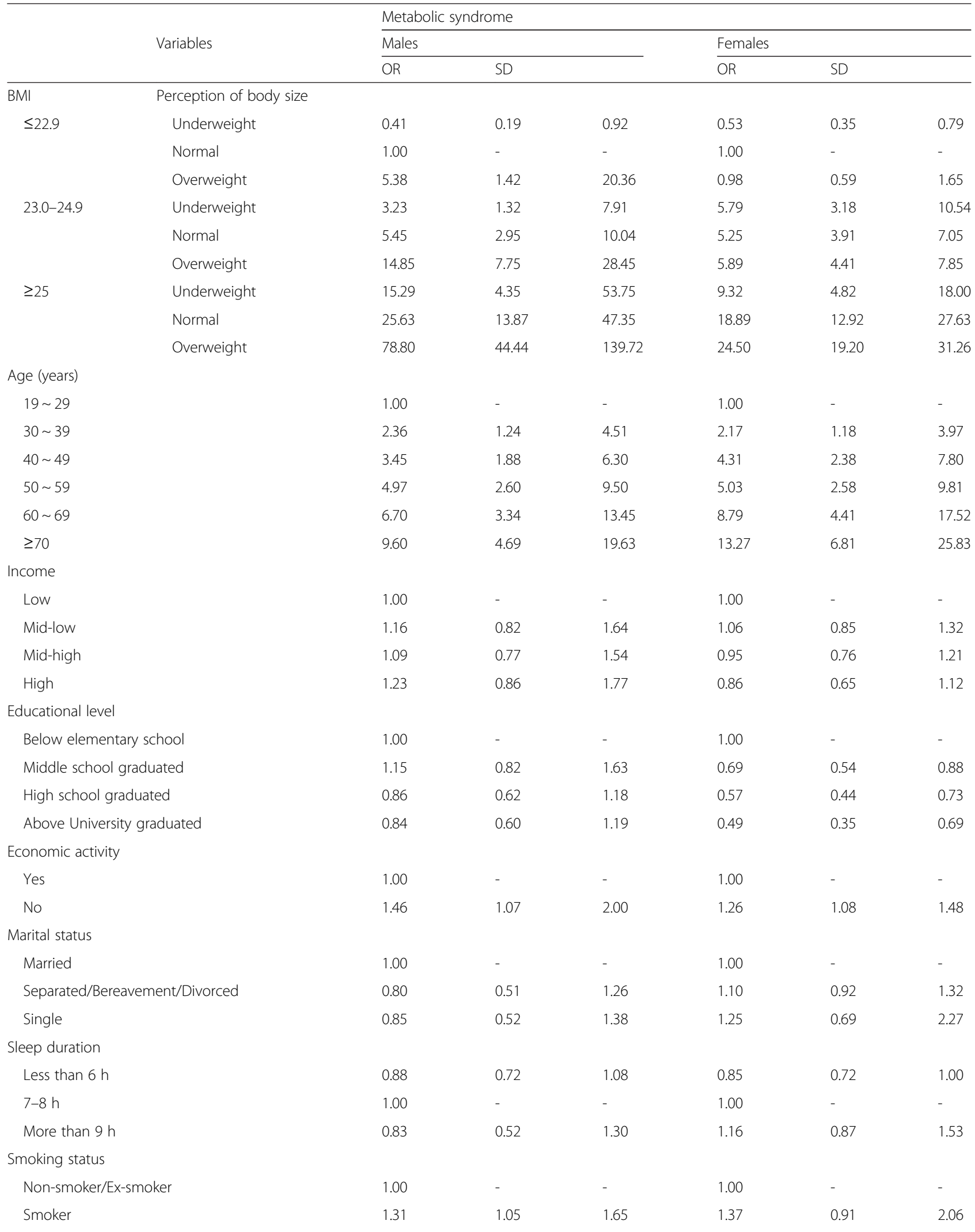


Table 5 Results of multivariable logistic regression analysis for the relationship between BMI/body size perception and metabolic syndrome (Continued)

\begin{tabular}{|c|c|c|c|c|c|c|}
\hline \multicolumn{7}{|l|}{ Alcohol consumption } \\
\hline Never & 1.00 & - & - & 1.00 & - & - \\
\hline Less than 1 time per month & 1.01 & 0.71 & 1.43 & 0.95 & 0.79 & 1.14 \\
\hline Less than 3 times per week & 1.12 & 0.81 & 1.54 & 0.98 & 0.79 & 1.22 \\
\hline More than 4 times per week & 1.91 & 1.33 & 2.73 & 0.83 & 0.48 & 1.41 \\
\hline \multicolumn{7}{|l|}{ Stress awareness } \\
\hline High & 0.93 & 0.66 & 1.31 & 0.90 & 0.70 & 1.17 \\
\hline Moderate & 0.87 & 0.66 & 1.15 & 0.85 & 0.68 & 1.06 \\
\hline Low & 1.00 & - & - & 1.00 & - & - \\
\hline \multicolumn{7}{|l|}{ Moderate physical activity } \\
\hline Yes & 1.00 & - & - & 1.00 & - & - \\
\hline No & 1.25 & 0.87 & 1.80 & 1.09 & 0.84 & 1.42 \\
\hline \multicolumn{7}{|l|}{ Survey year } \\
\hline 2010 & 1.00 & - & - & 1.00 & - & - \\
\hline 2011 & 1.12 & 0.87 & 1.43 & 0.90 & 0.74 & 1.09 \\
\hline 2012 & 0.84 & 0.64 & 1.10 & 1.15 & 0.95 & 1.38 \\
\hline \multicolumn{7}{|l|}{ Menopause } \\
\hline Not yet & - & - & - & 1.00 & - & - \\
\hline Yes & - & - & - & 1.46 & 1.09 & 1.94 \\
\hline Total energy intake & 1.00 & 0.99 & 1.01 & 1.01 & 0.99 & 1.02 \\
\hline C-statistics & $0.865^{*}$ & & & $0.877^{*}$ & & \\
\hline
\end{tabular}

$B M I$ body mass index, $O R$ odds ratio, $S D$ standard deviation

*P-value for likelihood ratio test $<0.05$

In accordance with this, we found that South Korean subjects with the same BMI exhibited different behaviors based on their body size perception; therefore, predicting metabolic syndrome risk solely based on BMI did not take different behaviors into account [26, 27].

Thus, our findings suggest that the combination of body size perception and BMI could be more useful in predicting the risk of metabolic syndrome than BMI alone. The use of complementary predictors could improve prediction and prognostication.

This study has several strengths compared to previous investigations. First, we used nationally representative data, so our study results are representative and generalizable to South Korea citizens. Such data are especially helpful in establishing evidence-based health policies. To our knowledge, this is the first attempt to study the relationship between the combined effect of BMI/body size perception and metabolic syndrome in South Korea, despite numerous issues regarding the management of these health issues in the country. Therefore, our findings should be helpful in identifying ways to address these critical issues.

Our study also has some limitations. First, due to the cross-sectional nature of the KNHANES, it is not possible to identify causal relationships. Other issues must be considered to more accurately measure the relationship between the combined effect of BMI/body size perception and metabolic syndrome. Next, our findings included high OR values, not general OR values. Further studies are needed to confirm our findings, which show a combined effect for metabolic syndrome in relatively small study populations (after stratification). Nevertheless, the overall trends of our findings have serious implications for the management of metabolic syndrome. Third, body size perception was measured by the subjects' answers to the question: "How do you perceive your body size?" The response could have been incorrectly perceived by researchers and is not a truly scientific measurement. Finally, our analysis did not include important details such as respondent food consumption. Thus, multiple variables that are not a major factor of metabolic syndrome were not considered in our findings.

Despite these limitations, our findings suggest that the combined effect of BMI and body size perception can be used to predict the presence of metabolic syndrome. Based on these findings, it is important for health policy makers to identify solutions for controlling metabolic syndrome. 
Table 6 Results of subgroup analysis for the relationship between combined effect of BMI/body size perception and metabolic syndrome by age group

\begin{tabular}{|c|c|c|c|c|c|c|c|c|c|c|c|c|c|}
\hline \multirow{4}{*}{\multicolumn{2}{|c|}{ Type of predictor for metabolic syndrome }} & \multicolumn{12}{|c|}{ Metabolic syndrome } \\
\hline & & \multicolumn{6}{|l|}{ Males } & \multicolumn{6}{|c|}{ Females } \\
\hline & & \multicolumn{3}{|c|}{ Less than 64 years } & \multicolumn{3}{|c|}{ More than 65 years } & \multicolumn{3}{|c|}{ Less than 64 years } & \multicolumn{3}{|c|}{ More than 65 years } \\
\hline & & \multirow[t]{2}{*}{$\mathrm{OR}$} & \multicolumn{2}{|l|}{ SD } & \multirow[t]{2}{*}{ OR } & \multicolumn{2}{|l|}{ SD } & \multirow[t]{2}{*}{$\mathrm{OR}$} & \multicolumn{2}{|l|}{ SD } & \multirow[t]{2}{*}{ OR } & \multicolumn{2}{|l|}{ SD } \\
\hline$\overline{\mathrm{BMI}}$ & Perception of body size & & & & & & & & & & & & \\
\hline$\leq 22.9$ & - & 1.00 & - & - & 1.00 & - & - & 1.00 & - & - & 1.00 & - & - \\
\hline $23.0-24.9$ & - & 9.13 & 4.87 & 17.12 & 10.74 & 6.00 & 19.21 & 9.13 & 6.46 & 12.88 & 5.32 & 4.04 & 7.02 \\
\hline$\geq 25$ & - & 74.63 & 42.15 & 132.16 & 55.07 & 30.98 & 97.88 & 39.26 & 28.46 & 54.15 & 15.78 & 11.77 & 21.15 \\
\hline \multirow[t]{3}{*}{$\leq 22.9$} & Underweight & 0.39 & 0.12 & 1.28 & 0.41 & 0.13 & 1.32 & 0.47 & 0.19 & 1.13 & 0.40 & 0.25 & 0.64 \\
\hline & Normal & 1.00 & - & - & 1.00 & - & - & 1.00 & - & - & 1.00 & - & - \\
\hline & Overweight & 5.29 & 1.04 & 26.88 & 2.46 & 0.43 & 14.13 & 1.15 & 0.60 & 2.20 & 1.25 & 0.47 & 3.38 \\
\hline \multirow[t]{3}{*}{$23.0-24.9$} & Underweight & 0.97 & 0.17 & 5.73 & 6.10 & 1.97 & 18.95 & 21.35 & 5.80 & 78.56 & 2.75 & 1.58 & 4.80 \\
\hline & Normal & 4.81 & 1.95 & 11.89 & 6.97 & 3.36 & 14.46 & 7.02 & 4.37 & 11.27 & 3.54 & 2.36 & 5.31 \\
\hline & Overweight & 15.32 & 6.06 & 38.74 & 12.20 & 4.77 & 31.22 & 7.96 & 5.13 & 12.37 & 4.44 & 2.59 & 7.60 \\
\hline \multirow[t]{3}{*}{$\geq 25$} & Underweight & 23.41 & 3.74 & 146.52 & 7.82 & 1.38 & 44.43 & 10.18 & 2.27 & 45.72 & 6.52 & 3.14 & 13.53 \\
\hline & Normal & 26.09 & 10.87 & 62.63 & 25.64 & 11.60 & 56.68 & 24.44 & 13.40 & 44.56 & 13.81 & 8.50 & 22.44 \\
\hline & Overweight & 75.93 & 33.14 & 173.96 & 59.06 & 28.24 & 123.50 & 36.60 & 24.83 & 53.95 & 10.13 & 6.97 & 14.73 \\
\hline \multirow[t]{2}{*}{ C-statistics } & Only BMI Model & $0.846^{*}$ & & & $0.856^{*}$ & & & $0.877^{*}$ & & & $0.784^{*}$ & & \\
\hline & Combination Model & $0.858^{*}$ & & & $0.864^{*}$ & & & $0.882^{*}$ & & & $0.791^{*}$ & & \\
\hline
\end{tabular}


However, further studies of those issues are needed to establish an effective strategy.

\section{Conclusion}

The combined effects of body size perception and BMI affect the risk for metabolic syndrome in individuals with the same BMI. Our findings suggest that both variables should be used in predicting the risk of disease to reduce risk of inaccurate predictions.

\section{Abbreviations}

OECD: Organization for Economic Co-operation and Development: HDL: High-density lipoprotein; BMl: Body mass index; KNHANES: Korea National Health and Nutrition Examination Surveys; KCDC: Korea Centers for Disease Control and Prevention; IDF: International Diabetes Federation; OR: Odds ratio; SD: Standard deviation.

\section{Competing interests}

The authors declare that they have no competing interests.

\section{Authors' contributions}

SHY and KTH led the design and conception of the study, performed the data analysis, and wrote the manuscript. SJK, TYS, BJ, and ECP participated in the study design and provided intellectual input for the development of the manuscript. WRK provided re-editing for our manuscript to improve quality of scientific writing. ECP helped draft this manuscript. All authors read and approved the final manuscript.

\section{Author details}

${ }^{1}$ Department of Health Policy and Management, Graduate School of Public Health, Yonsei University, Seoul, Republic of Korea. ${ }^{2}$ Department of Public Health, Graduate School, Yonsei University, Seoul, Republic of Korea. ${ }^{3}$ Institute of Health Services Research, Yonsei University College of Medicine Seoul, Republic of Korea. ${ }^{4}$ Department of Health Administration, Namseoul University, Cheonan, Republic of Korea. ${ }^{5}$ Department of Health Services Administration, Yuhan University, Bucheon, Republic of Korea. ${ }^{6}$ Department of Preventive Medicine, Yonsei University College of Medicine, Seoul, Republic of Korea.

Received: 24 February 2015 Accepted: 13 May 2015

Published online: 17 June 2015

\section{References}

1. Kim NS, Moon OR, Kang JH, Lee SY, Jeong BG, Lee SJ, et al. Increasing prevalence of obesity related disease for Koreans associated with overweight and obesity. Korean J Prev Med. 2001;34(4):309-15.

2. Abegunde DO, Mathers CD, Adam T, Ortegon M, Strong K. The burden and costs of chronic diseases in low-income and middle-income countries. Lancet. 2007;370(9603):1929-38.

3. Statistics Korea. All causes of mortality. 2013.

4. Organization for Economic Cooperation and Development. Health at a Glance 2013: OECD Indicators. 2013.

5. Isomaa B, Almgren P, Tuomi T, Forsén B, Lahti K, Nissen M, et al. Cardiovascular morbidity and mortality associated with the metabolic syndrome. Diabetes Care. 2001;24(4):683-9.

6. Kahn R, Buse J, Ferrannini E, Stern M. The metabolic syndrome: time for a critical appraisal Joint statement from the American Diabetes Association and the European Association for the Study of Diabetes. Diabetes Care. 2005;28(9):2289-304.

7. Lim S, Shin H, Song JH, Kwak SH, Kang SM, Yoon JW, et al. Increasing prevalence of metabolic syndrome in Korea the Korean national health and nutrition examination survey for 1998-2007. Diabetes Care. 2011;34(6):1323-8.

8. Alberti K, Zimmet P, Shaw J. Metabolic syndrome-a new world-wide definition. A consensus statement from the international diabetes federation. Diabet Med. 2006;23(5):469-80.

9. Flegal KM, Carroll MD, Kuczmarski RJ, Johnson CL. Overweight and obesity in the United States: prevalence and trends, 1960-1994. Int J Obes Relat Metab Disord. 1998;22(1):39-47
10. Ervin RB. Prevalence of metabolic syndrome among adults 20 years of age and over, by sex, age, race and ethnicity, and body mass index: United States. Natl Health Stat Report. 2009;13:1-8.

11. Lee CMY, Huxley RR, Wildman RP, Woodward M. Indices of abdominal obesity are better discriminators of cardiovascular risk factors than BMI: a meta-analysis. J Clin Epidemiol. 2008;61(7):646-53.

12. Dietz WH, Bellizzi MC. Introduction: the use of body mass index to assess obesity in children. Am J Clin Nutr. 1999;70(1):123s-5.

13. Pham DD, Ku B, Shin C, Cho NH, Cha S, Kim JY. Thoracic-to-hip circumference ratio as a novel marker of type 2 diabetes, independent of body mass index and waist-to-hip ratio, in Korean adults. Diabetes Res Clin Pract. 2014;104(2):273-80.

14. Wright EJ, Whitehead TL. Perceptions of body size and obesity: a selected review of the literature. J Community Health. 1987;12(2-3):117-29.

15. KIM O, KIM K. Comparisons of body mass index, perception of body weight, body shape satisfaction, and self-esteem among Korean adolescents. Percept Mot Skills. 2003;97(3f):1339-46.

16. Park HS, Oh SW, Cho S-I, Choi WH, Kim YS. The metabolic syndrome and associated lifestyle factors among South Korean adults. Int J Epidemiol. 2004;33(2):328-36.

17. Park Y-W, Zhu S, Palaniappan L, Heshka S, Carnethon MR, Heymsfield SB. The metabolic syndrome: prevalence and associated risk factor findings in the US population from the Third National Health and Nutrition Examination Survey, 1988-1994. Arch Intern Med. 2003;163(4):427-36.

18. Lakka TA, Laaksonen DE, Lakka H-M, Männikkö N, Niskanen LK, Rauramaa R, et al. Sedentary lifestyle, poor cardiorespiratory fitness, and the metabolic syndrome. Med Sci Sports Exerc. 2003;35(8):1279-86.

19. Janssen I, Katzmarzyk PT, Ross R. Waist circumference and not body mass index explains obesity-related health risk. Am J Clin Nutr. 2004;79(3):379-84.

20. Stice $E$, Shaw HE. Role of body dissatisfaction in the onset and maintenance of eating pathology: A synthesis of research findings. J Psychosom Res. 2002;53(5):985-93.

21. Sakamaki R, Amamoto R, Mochida Y, Shinfuku N, Toyama K. A comparative study of food habits and body shape perception of university students in Japan and Korea. Nutr J. 2005;4(1):31.

22. Schulken ED, Pinciaro PJ, Sawyer RG, Jensen JG, Hoban MT. Sorority women's body size perceptions and their weight-related attitudes and behaviors. J Am Coll Health. 1997;46(2):69-74.

23. Zhu S, St-Onge M-P, Heshka S, Heymsfield SB. Lifestyle behaviors associated with lower risk of having the metabolic syndrome. Metabolism. 2004;53(11):1503-11.

24. Hu FB, Manson JE, Stampfer MJ, Colditz G, Liu S, Solomon CG, et al. Diet, lifestyle, and the risk of type 2 diabetes mellitus in women. $\mathrm{N}$ Engl J Med. 2001;345(11):790-7.

25. Paffenbarger Jr RS, Hyde RT, Wing AL, Lee I-M, Jung DL, Kampert JB. The association of changes in physical-activity level and other lifestyle characteristics with mortality among men. N Engl J Med. 1993;328(8):538-45.

26. Kim M, Lee $\mathrm{H}$. Overestimation of own body weights in female university students: associations with lifestyles, weight control behaviors and depression. Nutr Res Pract. 2010;4(6):499-506.

27. Kwak H-K, Lee MY, Kim MJ. Comparisons of body image perception, health related lifestyle and dietary behavior based on the self-rated health of university students in Seoul. Korean J Community Nutr. 2011;16(6):672-82.

\section{Submit your next manuscript to BioMed Central and take full advantage of:}

- Convenient online submission

- Thorough peer review

- No space constraints or color figure charges

- Immediate publication on acceptance

- Inclusion in PubMed, CAS, Scopus and Google Scholar

- Research which is freely available for redistribution 